\title{
An Analytical Insight into the Buckling Paradox for Circular Cylindrical Shells under Axial and Lateral Loading
}

\author{
Rabee Shamass, ${ }^{1}$ Giulio Alfano, ${ }^{1}$ and Federico Guarracino ${ }^{2}$ \\ ${ }^{1}$ Department of Mechanical, Aerospace and Civil Engineering, Brunel University, Uxbridge UB8 3PH, UK \\ ${ }^{2}$ Department of Structural Engineering, University of Naples "Federico II", Via Claudio 21, 80125 Naples, Italy \\ Correspondence should be addressed to Federico Guarracino; fguarrac@unina.it
}

Received 23 December 2014; Accepted 28 May 2015

Academic Editor: Xin-Lin Gao

Copyright (C) 2015 Rabee Shamass et al. This is an open access article distributed under the Creative Commons Attribution License, which permits unrestricted use, distribution, and reproduction in any medium, provided the original work is properly cited.

\begin{abstract}
A large number of authors in the past have concluded that the flow theory of plasticity tends to overestimate significantly the buckling load for many problems of plates and shells in the plastic range, while the deformation theory generally provides much more accurate predictions and is consequently used in practical applications. Following previous numerical studies by the same authors focused on axially compressed cylinders, the present work presents an analytical investigation which comprises the broader and different case of nonproportional loading. The analytical results are discussed and compared with experimental and numerical findings and the reason for the apparent discrepancy on the basis of the so-called "buckling paradox" appears once again to lay in the overconstrained kinematics on the basis of the analytical and numerical approaches present in the literature.
\end{abstract}

\section{Introduction}

Plastic buckling generally takes place in the case of moderately thick cylinders subjected to axial compression, external pressure, or torsion, alone or in combination, and has been largely investigated.

The plasticity models that have been proposed for metals in the strain hardening range for the study of plastic buckling can be divided into two groups: the "deformation theory" of plasticity and the "flow theory" of plasticity. In both of these theories the plastic deformation is a function of the second invariant of the deviatoric part of the stress tensor, given that volume changes in the plastic range are not permitted. The difference lies in the fact that in the deformation theory of plasticity it is assumed that the state of stress is uniquely determined by the state of strain as it would happen in any pathindependent nonlinear elastic constitutive law. Therefore, after a strain reversal, instead of recovering the initial elastic stiffness, the initial loading curve is followed. This behaviour is in contrast with the usual findings from experimental tests. The flow theory of plasticity, on the contrary, assumes that the increment of strain/stress is uniquely determined by the existing stress/strain and its increment. This assumption gives origin to a path-dependent relationship and the current stress depends not only on the value of the actual total strain but also on how this value has been reached.

As a consequence, notwithstanding its mathematical advantages, the deformation theory of plasticity is considered to lack somehow physical rigour in comparison to the flow theory $[1,2]$. Surprisingly, the employment of the deformation theory has been reported to predict buckling loads that are smaller than those obtained with the flow theory and in better accordance with the experimental tests. This fact has been often designated as the "plastic buckling paradox."

Recently the present authors have started to investigate the plastic buckling paradox by conducting accurate finiteelement modelling of buckling of cylindrical shells using both the flow theory and the deformation theory of plasticity $[3,4]$. Contrary to the common belief, they showed that, by using an accurate and carefully validated geometrically nonlinear finite element modelling, a very good agreement between numerical and experimental results can be obtained also in the case of the physically sound flow theory of plasticity. Consequently, according to the performed numerical investigations in the case of axially loaded cylinders, it can be affirmed that no plastic buckling paradox actually exists. Additionally, the flow theory of plasticity, which provides a physically sound description of the behaviour of metals, 
can even lead to predictions of the buckling stress which are in better agreement with the corresponding test results than those provided by use of the deformation theory, in contrast with the widely accepted belief that the flow theory leads to a significant overestimation of the buckling stress while the deformation theory leads to much more accurate predictions and, therefore, is the recommended choice for use in practical applications. On the basis of these numerical investigations, it was suggested that the roots of the discrepancy lie in the simplifying assumptions which have been regularly made with respect to the buckling modes and that the adoption of the deformation theory of plasticity simply results in counterbalancing the greater stiffness induced by kinematically constraining the cylinders to follow predefined buckling modes.

However, the case of axially loaded cylinders is relatively limited in order to draw general conclusions since in this case material points are generally subjected to proportional loading in the elastic range, and this remains relatively true also in the initial phase of plastic buckling. In fact, even more significant discrepancies are reported in the literature between the results of the flow and the deformation theories in the case of nonproportional loading.

Therefore the present investigation extends the analysis to the much more general case of nonproportional loading and, at the same time, makes use of an analytical treatment of the problem instead of the numerical one employed for the case of proportional loading. This makes it possible to analyse in detail the shape of the buckling modes both in the cases of the flow and of the deformation theory of plasticity.

The obtained analytical results are compared with the experimental and numerical results obtained in $[5,6]$ using the code BOSOR5 [7] and, for the purpose of validation, also with the numerical ones by the present authors [8].

Blachut et al. [5] conducted experimental and numerical analyses for 30 mild-steel machined cylinders, of different dimensions, subject to axial tension and increasing external pressure. Using the code BOSOR5 [7] for their numerical analyses they reported that the agreement between the two plasticity theories appeared strongly dependent on the diameter, $D$, and the length, $L$, of the cylindrical shell. For short cylinders $(D / L=1)$, the plastic-buckling pressure results predicted by the flow and deformation theories coincided only when the tensile axial load vanished [5]. By increasing the axial tensile load, the plastic buckling pressures predicted by the flow theory started to diverge quickly from those predicted by the deformation theory. Additionally, the flow theory failed to predict any buckling for high axial tensile load while tests confirmed the buckling occurrence. For specimens with length-to-diameter ratio $L / D$ ranging from 1.5 to 2.0 the results predicted by both theories were identical for a certain range of combined loading. However, for high values of applied tensile load, the predictions of the flow theory began to deviate from those of the deformation theory and became unrealistic in correspondence with large plastic strains.

Giezen et al. [6] conducted experiments and numerical analyses on two sets of tubes made of aluminium alloy 6061T4 and subjected to combined axial tension and external pressure in order to highlight the difference in buckling predictions of both the flow theory and the deformation theory again using the code BOSOR5. These tubes have $L / D$ ratios equal to one. In their test two different loading paths were considered. In the first one the axial tensile load was held constant and the external pressure was increased; in the second one, the external pressure was held constant and the axial tensile load was increased. Their numerical studies showed that the buckling pressure based on the flow theory increases with increasing applied tensile load while the experimental test revealed a reduction in buckling resistance with increasing axial tension. Thus as axial tension increased the discrepancy between test results and numerical results predicted by the flow theory also significantly increased. On the other hand, results predicted by the deformation theory displayed the same trend as in the test results. However, the deformation theory significantly underpredicted the buckling pressure observed experimentally for some loading paths. Therefore, Giezen [9] concluded that, generally speaking, both plasticity theories were unsuccessful in predicting buckling load.

For the case of cylinders subjected to axial tensile load and external pressure, Blachut et al. [5] and Giezen et al. [6] concluded that the flow theory significantly overpredicts the plastic strains and buckling loads for high tensile loads while deformation theory leads to acceptable plastic strains and buckling loads that are more in line with experimental observations in most cases. This moved researchers to attempt a revised deformation theory by including unloading [10] or propose a total deformation theory applicable for nonproportional loading defined as a sequence of linear loadings [11].

The analytical approach employed in this work moves from the formulation presented by Chakrabarty [12] and encompasses, differently from the original formulation of Chakrabarty, both the flow and deformation theories.

It is found that the plastic buckling results calculated analytically using both the flow and deformation theories closely match those, when available, obtained numerically by using the code BOSOR5 $[5,7]$. The analytical results thus confirm that the flow theory seems to overpredict buckling pressures for high values of applied tensile load while the deformation theory predictions appear to be in better agreement with experimental results.

However, going more in depth by means of the proposed analytical approach, it is possible to focus the attention on the buckling shapes and confirm, in such a way, that the root of the discrepancy lays in the assumed harmonic buckling modes along the circumference at the bifurcation.

In fact it is found that in the case of nonproportional loading the analytical and numerical approaches based on a certain class of harmonic buckling functions tend to overestimate the buckling loads when using the flow theory on account of a shift of the buckling modes. The deformation theory, on the contrary, tends to point to buckling shapes of lower order and thus counterbalances the excessive stiffness of the cylinder caused by the kinematic overconstraining.

In conclusion, when the buckling modes are the same, and in the case of nonproportional loading, the flow and 


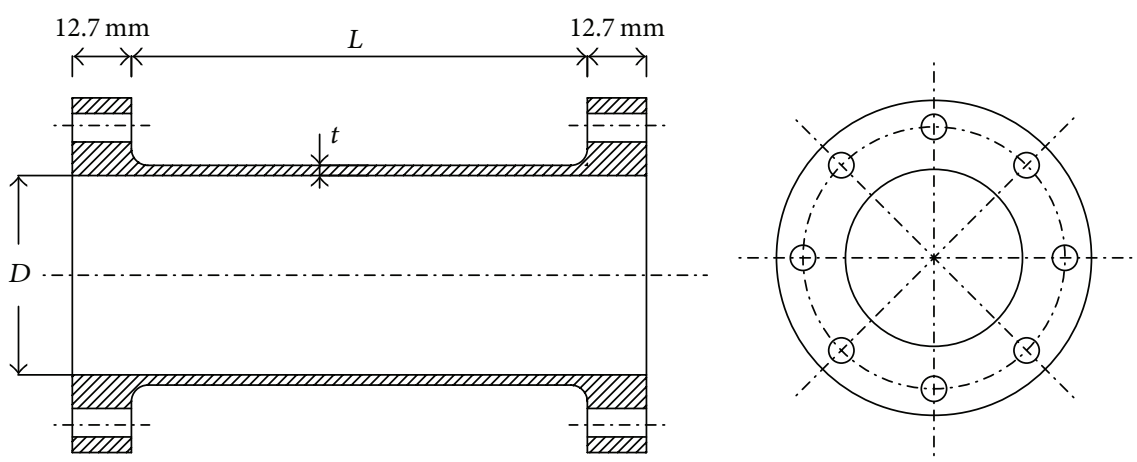

FIgURe 1: Experimental setting by Blachut et al. [5].

TABLE 1: Ramberg-Osgood parameters.

\begin{tabular}{|c|c|c|c|c|c|}
\hline & $E[\mathrm{MPa}]$ & $\sigma_{y}[\mathrm{MPa}]$ & $v$ & $n$ & $a$ \\
\hline Blachut et al. [5] & 212000 & 328 or 290 & 0.31 & 300 & 0.428 \\
\hline Giezen et al._-Set A [6] & 65129.73 & 177.75 & 0.3 & 16 & 0.733 \\
\hline Giezen et al._-Set B [6] & 60986.34 & 165.37 & 0.3 & 11.76 & 0.738 \\
\hline
\end{tabular}

deformation theory of plasticity provide the same buckling loads. It is worth recalling that the numerical FE approach [8], which is not kinematically overconstrained by a choice of predefined harmonic buckling modes, provides results which are in line with the experimental ones in the case of proportional loading.

As such, the conclusion of the present investigation is that also in the case of cylinders subjected to the more general and technically relevant case of nonproportional loading actually there seems to be no plastic buckling paradox.

\section{Experimental Data}

Blachut et al. [5] conducted tests on 30 machined cylinders made of mild steel with outer diameter $34 \mathrm{~mm}$ and lengthdiameter ratio $(L / D)$ of $1.0,1.5$, and 2.0. In the experimental setting, one flange of the specimen was rigidly attached to the end flange of the pressure chamber and the other flange was bolted to a coupling device which in turn was bolted to the load cell; see Figure 1.

In order to prevent any eccentricity of the axial load exerted on the specimen, the load cell was centered with respect to the test chamber. The authors pointed out that the maximum initial radial imperfections measured at the midlength of the specimens were about $1 \%$ of the thickness.

Giezen et al. [6] tested cylindrical specimens of aluminium alloy 6061-T4. Two sets of specimens were tested, namely, Set A and Set B. The average wall-thickness values of the first and second sets were 0.76 and $0.71 \mathrm{~mm}$, respectively, and the length-diameter ratio $(L / D)$ was equal to one. The maximum initial imperfection was found to be about $0.076 \mathrm{~mm}$ (10\% of the thickness).

Both authors applied two types of loading, that is, axial tensile load in the longitudinal direction and external lateral pressure. In this manner the buckling was investigated in the case of nonproportional loading.
Blachut et al. [5] and Giezen et al. [6] conducted longitudinal tensile tests on a number of coupons to determine the mechanical properties of the cylindrical specimens. For both tests the uniaxial stress-strain relationship of the material under monotonic loading can be characterised by the Ramberg-Osgood law

$$
\varepsilon=\frac{1}{E}\left[\sigma+\alpha\left(\frac{\sigma}{\sigma_{y}}\right)^{n-1} \sigma\right],
$$

where $\sigma$ and $\varepsilon$ denote uniaxial stress and strain, $E$ and $\nu$ are Young's modulus and Poisson's ratio, $\sigma_{y}$ is the nominal yield strength, $\alpha$ is the yield offset, and $n$ is the hardening parameter.

The Ramberg-Osgood parameters characterising the tests are reported in Table 1.

As it can be seen, for example, in the case of experiments by Giezen et al. [6] (Figure 2), the Ramberg-Osgood fit of the experimental data is very accurate.

\section{Analytical Treatment}

In order to analyse the results from the tests mentioned in the previous section, an analytical treatment of the buckling of a circular cylindrical shell under combined axial load and lateral pressure has been developed on the basis of the approach presented by Chakrabarty [12]. The analytical treatment has been extended to cover both the use of deformation and flow theories of plasticity.

For the sake of completeness, in the following, a brief review of the constitutive equations for both theories of plasticity is given.

3.1. Constitutive Relationships Based on the Deformation Theory of Plasticity. The deformation theory of plasticity is based on the assumption that for continued loading the state 


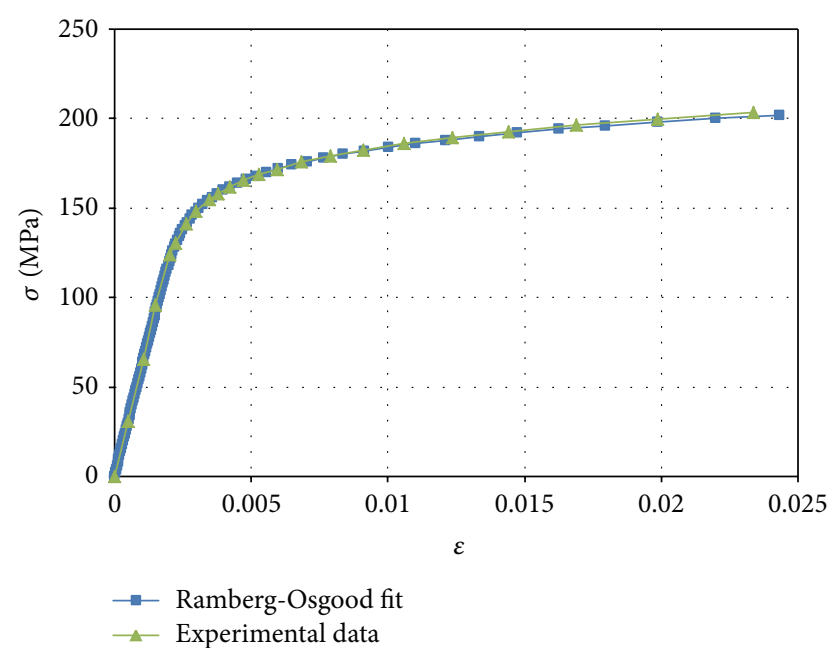

Figure 2: Ramberg-Osgood fit (Set B) [6].

of stress is uniquely determined by the state of strain and, therefore, it is a special class of path-independent nonlinear elasticity constitutive laws.

The constitutive relationship for the deformation theory of plasticity can be obtained by extending the RambergOsgood law to the case of a multiaxial stress state by means of the $J_{2}$ von Mises formulation and results in the following path-independent expression $[13,14]$ :

$$
\begin{aligned}
\boldsymbol{\varepsilon}= & (1+\nu) \operatorname{dev} \boldsymbol{\sigma}-(1-2 \nu) \operatorname{sph} \boldsymbol{\sigma} \\
& +\frac{3}{2} \alpha\left(\frac{\sqrt{3 / 2} \operatorname{dev} \boldsymbol{\sigma}}{\sigma_{y}}\right)^{n-1} \operatorname{dev} \boldsymbol{\sigma}
\end{aligned}
$$

where $\boldsymbol{\varepsilon}$ and $\boldsymbol{\sigma}$ denote the strain and stress tensors, while dev $\boldsymbol{\sigma}$ and sph $\sigma$ denote the deviatoric and spherical parts of the stress tensor, respectively.

Since the deformation theory of plasticity requires the same input values as the Ramberg-Osgood formula, the material constants of Table 1 have been directly employed for the present study.

3.2. Constitutive Relationships Based on the Flow Theory of Plasticity. The $J_{2}$ flow theory of plasticity in the small-strain regime with nonlinear isotropic hardening [14] is based on the additive decomposition of the spatial rate of the deformation tensor $\dot{\boldsymbol{\varepsilon}}$ into its elastic and plastic parts $\dot{\boldsymbol{\varepsilon}}_{e}$ and $\dot{\boldsymbol{\varepsilon}}_{p}:$ that is,

$$
\dot{\boldsymbol{\varepsilon}}=\dot{\boldsymbol{\varepsilon}}_{e}+\dot{\boldsymbol{\varepsilon}}_{p}
$$

and the rate of increment of the Cauchy stress tensor, $\dot{\boldsymbol{\sigma}}$, is given by the following isotropic linear elastic relationship:

$$
\dot{\boldsymbol{\sigma}}=2 G \dot{\boldsymbol{\varepsilon}}_{\mathrm{e}}+\lambda \operatorname{tr} \dot{\boldsymbol{\varepsilon}}_{\mathrm{e}} \mathbf{I}
$$

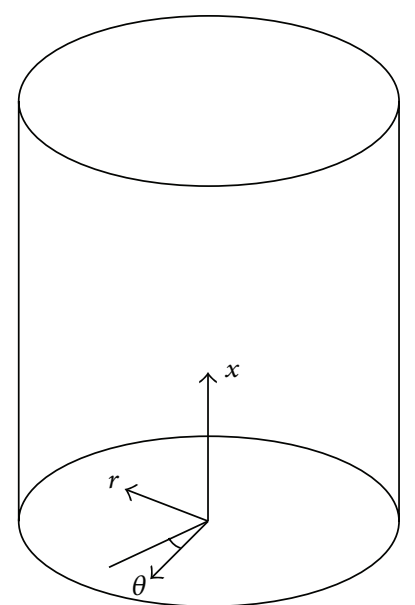

Figure 3: The cylindrical reference system.

where $G$ and $\lambda$ are Lamé's elastic constants and I is the identity tensor.

The plastic behaviour is introduced by means of the von Misses yield function, $f$,

$$
f\left(\boldsymbol{\sigma}, \boldsymbol{\varepsilon}_{p}^{\mathrm{eq}}\right)=\operatorname{dev} \boldsymbol{\sigma}-\sqrt{\frac{2}{3}} \overline{\boldsymbol{\sigma}}\left(\boldsymbol{\varepsilon}_{p}^{\mathrm{eq}}\right),
$$

where $\overline{\boldsymbol{\sigma}}$ represents the uniaxial yield strength. In order to describe the nonlinear isotropic hardening, $\bar{\sigma}$ is assumed to be an increasing function of the equivalent plastic strain $\boldsymbol{\varepsilon}_{p}^{\mathrm{eq}}$, defined at any time, $t$, as follows:

$$
\boldsymbol{\varepsilon}_{p}^{\mathrm{eq}}(t)=\int_{-\infty}^{\mathrm{t}}\left|\dot{\boldsymbol{\varepsilon}}_{p}(\tau)\right| \mathrm{d} \tau .
$$

The rate of the plastic strain is given by the associate flow rule; that is,

$$
\dot{\boldsymbol{\varepsilon}}_{p}=\dot{\eta}\left(\frac{\partial f}{\partial s}\right)_{s=\operatorname{dev} \sigma},
$$

where $\dot{\eta}$ is the plastic multiplier, satisfying the conditions

$$
\begin{aligned}
\dot{\eta} & \geq 0, \\
f\left(\boldsymbol{\sigma}, \boldsymbol{\varepsilon}_{p}^{\mathrm{eq}}\right) & \leq 0, \\
\dot{\eta} f\left(\boldsymbol{\sigma}, \boldsymbol{\varepsilon}_{p}^{\mathrm{eq}}\right) & =0 .
\end{aligned}
$$

3.3. Buckling Analysis. In order to determine the bifurcation load for a circular cylindrical shell with mean radius $R$, length $L$, and uniform thickness $h$, subject to combined axial tension and uniform external pressure, let $x$ be the axis of the shell and $r$ the radial direction. Then, the angle $\theta \in[0,2 \pi]$ defines a set of cylindrical coordinates for the shell; see Figure 3.

At any point within the shell $z$ is the distance of the point from the middle surface of the shell, which is taken positive if the point is on the outer side of the middle 
surface. The components of the velocity with respect to the above cylindrical coordinates at the considered point may be written as follows:

$$
\begin{aligned}
& v_{x}=u+z \omega_{\theta}, \\
& v_{\theta}=v-z \omega_{x}, \\
& v_{r}=w,
\end{aligned}
$$

where $u, v$, and $w$ denote the velocities at the middle surface in $x, \theta$, and radial directions, respectively, and $\omega_{x}$ and $\omega_{\theta}$ indicate the rotational velocities of the normal to the middle surface about the positive $x$ - and $\theta$-axes, respectively.

In the realm of the thin-shell theory, $\omega_{x}$ and $\omega_{\theta}$ are related to the midsurface velocities components as follows:

$$
\begin{aligned}
& \omega_{x}=\frac{1}{R}\left(\frac{\partial w}{\partial \theta}-v\right), \\
& \omega_{\theta}=-\frac{\partial w}{\partial x} .
\end{aligned}
$$

At the onset of bifurcation different modes of deformation can be found as a solution of the rate problem. A key assumption is to characterise such modes of deformation with the following harmonic expressions for $u, v$, and $w$ :

$$
\begin{gathered}
u=U \cos (\mu \xi) \sin (m \theta), \\
v=V \sin (\mu \xi) \cos (m \theta), \\
w=W \sin (\mu \xi) \sin (m \theta),
\end{gathered}
$$

where $U, V$, and $W$ are arbitrary constants, $\mu=n \pi R / L$, $\xi=x / R$, and $n$ and $m$ are two positive integers. $n$ represents the number of half-waves along the generator of the cylinder and $m$ denotes the number of waves in the circumferential direction.

The constitutive equations of the flow and deformation theories are based on the Jaumann stress rate as follows [12]:

$$
\begin{aligned}
& \dot{\sigma}_{x x}=\frac{E}{1+v}\left(\alpha \dot{\varepsilon}_{x x}+\beta \dot{\varepsilon}_{\theta \theta}\right), \\
& \dot{\sigma}_{\theta \theta}=\frac{E}{1+v}\left(\beta \dot{\varepsilon}_{x x}+\gamma \dot{\varepsilon}_{\theta \theta}\right), \\
& \dot{\tau}_{x \theta}=\frac{E}{1+v}\left(\frac{2+2 v}{E} G \dot{\varepsilon}_{x \theta}\right),
\end{aligned}
$$

where $E$ is the elastic modulus, $G$ is the effective shear modulus, and $v$ is the Poisson ratio for the material.
In the present case, for the case of the flow theory based on Prandtl-Reuss equations, it is

$$
\begin{aligned}
& \alpha=\frac{1+\nu}{\rho}\left[4-3\left(1-\frac{E_{t}}{E}\right) \frac{\sigma_{x x}^{2}}{\bar{\sigma}^{2}}\right] \\
& \beta=\frac{1+\nu}{\rho}\left[2-2(1-2 \nu) \frac{E_{t}}{E}-3\left(1-\frac{E_{t}}{E}\right) \frac{\sigma_{x x} \sigma_{\theta \theta}}{\bar{\sigma}^{2}}\right] \\
& \gamma=\frac{1+\nu}{\rho}\left[4-3\left(1-\frac{E_{t}}{E}\right) \frac{\sigma_{\theta \theta}^{2}}{\bar{\sigma}^{2}}\right] \\
& \rho=(5-4 \nu)-(1-2 \nu)^{2} \frac{E_{t}}{E} \\
& G=\frac{E}{2(1+\nu)} .
\end{aligned}
$$

On the other hand, for the case of the deformation theory based on Hencky equations, it is

$$
\begin{aligned}
\alpha & =\frac{1+\nu}{\rho}\left[4-3\left(1-\frac{E_{t}}{E_{s}}\right) \frac{\sigma_{x x}^{2}}{\bar{\sigma}^{2}}\right], \\
\beta & =\frac{1+\nu}{\rho}\left[2-2(1-2 \nu) \frac{E_{t}}{E}-3\left(1-\frac{E_{t}}{E_{s}}\right) \frac{\sigma_{x x} \sigma_{\theta \theta}}{\bar{\sigma}^{2}}\right], \\
\gamma & =\frac{1+\nu}{\rho}\left[4-3\left(1-\frac{E_{t}}{E_{s}}\right) \frac{\sigma_{\theta \theta}^{2}}{\bar{\sigma}^{2}}\right], \\
\rho & =3 \frac{E}{E_{s}}+(1-2 \nu)\left[2-(1-2 \nu) \frac{E_{t}}{E}\right. \\
& \left.-3\left(1-\frac{E_{t}}{E_{s}}\right) \frac{\sigma_{x x} \sigma_{\theta \theta}}{\bar{\sigma}^{2}}\right] \\
G & =\frac{E}{2(1+\nu)+3\left(E / E_{s}-1\right)} .
\end{aligned}
$$

Since the material obeys the von-Misses yield criterion, the effective stress $\bar{\sigma}$ is written, under the assumption of plane stress (i.e., $\sigma_{r r}=\tau_{z r}=\tau_{z \theta}=0$ ), as follows:

$$
\bar{\sigma}^{2}=\sigma_{x x}^{2}-\sigma_{x x} \sigma_{\theta \theta}+\sigma_{\theta \theta}^{2}
$$

Setting $\sigma_{x x}=\sigma_{t}$ and $\sigma_{\theta \theta}=-P R / h$ at the point of bifurcation, it is

$$
\bar{\sigma}^{2}=\sigma_{t}^{2}+\sigma_{t} \frac{P R}{h}+\left(\frac{P R}{h}\right)^{2} .
$$


The ratios of the elastic modulus $E$ to the tangent modulus, $E_{t}$, and to the secant modulus, $E_{s}$, are expressed by the Ramberg and Osgood relationship as

$$
\begin{aligned}
& \frac{E}{E_{t}}=1+\frac{\alpha n \bar{\sigma}^{n-1}}{\sigma_{y}{ }^{n-1}}, \\
& \frac{E}{E_{s}}=1+\frac{\alpha}{\sigma_{y}{ }^{n-1}} \bar{\sigma}^{n-1} .
\end{aligned}
$$

Under the assumption that the cylinders are simply supported at both ends and following the same line of reasoning as in [12], the equilibrium equations at the bifurcation point lead to the following set of buckling equations in the unknown constants $U, V$, and $W$ :

$$
\begin{aligned}
& {\left[\alpha \mu^{2}+\left(\frac{1+v}{E} G-q\right) m^{2}\right] U-\left(\frac{1+v}{E} G+\beta\right) \mu m V} \\
& -(\beta+q) \mu W=0, \\
& -\left(\frac{1+v}{E} G+\beta\right) \mu m U+\left[\left(\frac{1+v}{E} G \mu^{2}+\gamma m^{2}+\mu^{2} s\right)\right. \\
& \left.\quad+k\left(\frac{4+4 v}{E} G \mu^{2}+\gamma m^{2}\right)\right] V+[\gamma m \\
& \left.\quad+k m\left\{\left(\frac{4+4 v}{E} G+\beta\right) \mu^{2}+\gamma m^{2}\right\}\right] W=0, \\
& -(\beta+q) \mu U+\{\gamma m \\
& \left.\quad+k m\left[\left(\frac{4+4 \nu}{E} G+\beta\right) \mu^{2}+\gamma m^{2}\right]\right\} V+\left\{\gamma+\mu^{2} s\right. \\
& \quad-\left(m^{2}-1\right) q \\
& \left.+k\left[\alpha \mu^{4}+\left(\frac{4+4 \nu}{E} G+2 \beta\right) \mu^{2} m^{2}+\gamma m^{4}\right]\right\} W \\
& \quad+0,
\end{aligned}
$$

and a sufficient condition for bifurcation to take place is that the following characteristic equation is satisfied:

$$
A+B k=C s+D q
$$

where $s$ and $q$ are related to the applied average axial stress and external pressure, respectively, and $k$ is a geometrydependent parameter as follows:

$$
\begin{aligned}
& s=(1+v) \frac{\sigma_{t}}{E}, \\
& q=(1+v) \frac{P R}{E h}, \\
& k=\frac{h^{2}}{12 R^{2}} .
\end{aligned}
$$

It is worth noticing that (20) is obtained by neglecting the higher-order terms which involve the square and products of $s, q$, and $k . A, B, C$, and $D$ are obtained in such a way that
(20) is valid for the case of combined axial tensile stress and external pressure and the use of both flow and deformation theories of plasticity.

It is

$$
\begin{aligned}
A & =\frac{G \mu^{4}}{E}\left(-\beta^{2}+\alpha \gamma-\beta^{2} \nu+\alpha \gamma \nu\right), \\
B= & \frac{1}{E^{2}}\left\{-E^{2} m^{2}\left(\beta^{2}-\alpha \gamma\right) \mu^{2}\left[\left(-1+m^{2}\right)^{2} \gamma\right.\right. \\
& \left.+2\left(-1+m^{2}\right) \beta \mu^{2}+\alpha \mu^{4}\right]+E G(1+\nu)\left[m^{4}(-1\right. \\
& \left.+m^{2}\right)^{2} \gamma^{2}+2\left(\left(-2+5 m^{2}-4 m^{4}\right) \beta^{2}\right. \\
& \left.\left.+\left(2-4 m^{2}+3 m^{4}\right) \alpha \gamma\right) \mu^{4}+\alpha^{2} \mu^{8}\right]+4 G^{2} m^{2} \mu^{2}(1 \\
& \left.+\nu)^{2}\left[\left(-1+m^{2}\right)^{2} \gamma-2\left(-1+m^{2}\right) \beta \mu^{2}+\alpha \mu^{4}\right]\right\} \\
C & =-\frac{\mu^{2}}{E}\left\{E\left(-1+m^{2}\right)\left(\beta^{2}-\alpha \gamma\right) \mu^{2}+G(1+\nu)\right. \\
& \left.\cdot\left[m^{4} \gamma+\alpha \mu^{4}+m^{2}\left(\gamma-2 \beta \mu^{2}\right)\right]\right\}, \\
D & =-\frac{1}{E}\left\{E m^{2}\left(-1+m^{2}\right)\left(\beta^{2}-\alpha \gamma\right) \mu^{2}-G(1+\nu)\right. \\
& \cdot\left[m^{6} \gamma-(\alpha-2 \beta) \mu^{4}+m^{2} \mu^{2}\left(2 \beta-\gamma+\alpha \mu^{2}\right)\right. \\
& \left.\left.-m^{4}\left(\gamma+2 \beta \mu^{2}\right)\right]\right\} .
\end{aligned}
$$

From (20) it follows that

$$
q=\frac{(A+B k-C s)}{D}
$$

Consequently, for sequential values of $m$ and $n$, a series of corresponding values of the external pressure, $P$, can be obtained. The smallest value of $P$ and the corresponding determinations of $m$ and $n$ provide the buckling pressure and the corresponding buckling mode.

\section{Results and Discussion}

Using the Ramberg-Osgood input parameters reported in Table 1, the buckling pressures and corresponding buckling modes have been analytically calculated using (23) and reported in Table 3, using both the flow and deformation theories. The smallest eigenvalue in all the examined cases corresponds to $n=1$, which means that only one half-wave is formed in the longitudinal direction of the cylinder axis.

Table 2 collects the results from a subset of experimental tests and BOSOR5 numerical analyses $[5,6]$. The results have been chosen to represent cases in which the flow theory of plasticity, according to BOSOR5, does not provide a buckling load or strongly overestimates the ones from tests and cases in which there is agreement between the flow and deformation theory of plasticity. 
TABLE 2: Experimental versus BOSOR5 results (NA = not available).

\begin{tabular}{|c|c|c|c|c|c|c|c|}
\hline \multirow[b]{2}{*}{ Sp. } & \multicolumn{3}{|c|}{ Experimental results } & \multicolumn{2}{|c|}{ BOSOR5 results: deformation theory } & \multicolumn{2}{|c|}{ BOSOR5 results: flow theory } \\
\hline & $\begin{array}{l}\text { Number of } \\
\text { waves }\end{array}$ & $\begin{array}{l}\text { Axial tension } \\
(\mathrm{N})\end{array}$ & $\begin{array}{c}\text { External } \\
\text { pressure } \\
(\mathrm{MPa})\end{array}$ & $\begin{array}{l}\text { Number of } \\
\text { waves }\end{array}$ & $\begin{array}{c}\text { Buckling } \\
\text { pressure } \\
(\mathrm{MPa})\end{array}$ & $\begin{array}{l}\text { Number of } \\
\text { waves }\end{array}$ & $\begin{array}{c}\text { Buckling } \\
\text { pressure } \\
(\mathrm{MPa})\end{array}$ \\
\hline S1 & NA & 17960 & 4.07 & NA & 5.65 & NA & NA \\
\hline $\mathrm{S} 2$ & NA & 0 & 12.76 & NA & 13.29 & NA & 13.15 \\
\hline S5 & NA & 12010 & 8.28 & NA & 8.63 & NA & NA \\
\hline M2 & NA & 10670 & 8.14 & NA & 7.75 & NA & NA \\
\hline SP.1-Set B & 4 & 0 & 5.26 & 5 & 5.98 & 5 & 6.22 \\
\hline SP.6-Set B & 4 & 11771 & 3.00 & 5 & 3.32 & 4 & 6.20 \\
\hline SP.3-Set A & 5 & 2341 & 6.27 & 5 & 6.25 & 4 & 6.49 \\
\hline
\end{tabular}

TABLE 3: Numerical versus analytical results.

\begin{tabular}{|c|c|c|c|c|c|c|c|c|}
\hline \multirow[b]{2}{*}{ Sp. } & \multicolumn{2}{|c|}{$\begin{array}{c}\text { Numerical results } \\
\text { (ABAQUS): deformation } \\
\text { theory }\end{array}$} & \multicolumn{2}{|c|}{$\begin{array}{l}\text { Numerical results } \\
\text { (ABAQUS): flow theory }\end{array}$} & \multicolumn{2}{|c|}{$\begin{array}{l}\text { Analytical results: } \\
\text { deformation theory }\end{array}$} & \multicolumn{2}{|c|}{$\begin{array}{l}\text { Analytical results: flow } \\
\text { theory }\end{array}$} \\
\hline & $\begin{array}{l}\text { Number of } \\
\text { waves }\end{array}$ & $\begin{array}{l}\text { Buckling } \\
\text { pressure } \\
(\mathrm{MPa})\end{array}$ & $\begin{array}{l}\text { Number of } \\
\text { waves }\end{array}$ & $\begin{array}{l}\text { Buckling } \\
\text { pressure } \\
(\mathrm{MPa})\end{array}$ & $\begin{array}{l}\text { Number of } \\
\text { waves }\end{array}$ & $\begin{array}{l}\text { Buckling } \\
\text { pressure } \\
(\mathrm{MPa})\end{array}$ & $\begin{array}{l}\text { Number of } \\
\text { waves }\end{array}$ & $\begin{array}{l}\text { Buckling } \\
\text { pressure } \\
(\mathrm{MPa})\end{array}$ \\
\hline S1 & 4 & 5.53 & 4 & 5.64 & 4 & 5.29 & 2 & 16.24 \\
\hline S2 & 6 & 13.14 & 6 & 13.15 & 4 & 13.24 & 4 & 13.28 \\
\hline S5 & 4 & 8.73 & 4 & 8.83 & 4 & 8.56 & 2 & 11.02 \\
\hline M2 & 3 & 7.84 & 3 & 7.87 & 4 & 7.75 & 4 & 7.91 \\
\hline SP.1-Set B & 4 & 5.09 & 4 & 5.15 & 5 & 5.32 & 5 & 5.44 \\
\hline SP.6-Set B & 4 & 2.91 & 4 & 3.22 & 4 & 2.75 & 3 & 5.36 \\
\hline SP.3-Set A & 5 & 5.25 & 5 & 5.28 & 4 & 6.00 & 4 & 6.27 \\
\hline
\end{tabular}

Table 3, along with the analytical results from the presented treatment, shows the results from nonlinear FE analyses obtained by means of the commercial package ABAQUS $[8,13]$.

It is immediate to notice that the analytical treatment, differently from BOSOR5, always provides a value of the buckling pressure, albeit sometimes very different from the experimental results.

This is not surprising, given that the difference between the two theories lies, in the proposed procedure, only in the adoption of different values for the expressions of $\alpha, \beta$, and $\gamma$, (13) and (14), respectively.

On the contrary, the bifurcation load and the corresponding buckling mode are determined in BOSOR5 through a sequence of two subsequent analyses [7]. The first is a nonlinear prebuckling analysis which is valid for small strains and moderately large rotations and accounts for material nonlinearity. This nonlinear problem is solved using a strategy in which nested iteration loops are applied at each load level. The inner loop is used to resolve the nonlinear behaviour caused by the moderate large displacements using the Newton-Raphson method, and the outer loop is used to calculate the constitutive matrix and the plastic strain components. Loading and unloading status are checked iteratively by means of a subincremental strategy. The results from this first step are then used for the eigenvalue analysis. It is important to point out that BOSOR5 applies a discretization only to the longitudinal direction because the displacements are axisymmetric in the prebuckling phase and the buckling mode is assumed to vary harmonically in the circumferential direction.

As a result, BOSOR5 routines do not point to a buckling load in the cases of specimens S1, S2, S5, and M2 tested by Blachut et al. [5].

The numerical analyses conducted by means of the nonlinear FE code ABAQUS [8, 13], instead, lead to the correct determination of the buckling loads, in accordance with the experimental results, both for the deformation and the flow theory of plasticity.

The main finding from the presented analytical treatment is that when the buckling modes coincide using either the deformation or the flow theory, that is, in the case of specimens S2, M2, SP.1-Set B, and SP.3-Set A, the buckling loads are the same and in line with the experimental and FE results. When the buckling modes do not coincide in the case of the deformation or of the flow theory of plasticity, then the buckling loads provided by the flow theory of plasticity are much higher than those provided by the deformation theory; see specimens S1, S5, and SP.6-Set B.

It is worth pointing out that the buckling modes yielded by the presented analytical analysis do not need to coincide with those by the FE analyses or by the experimental results 


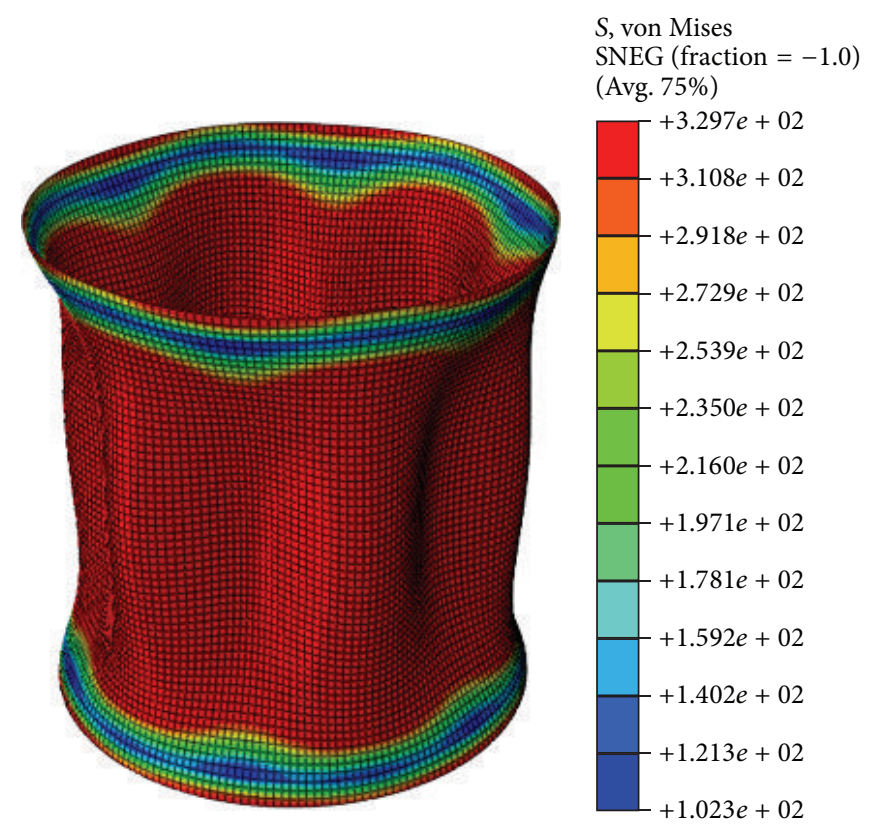

(a) Specimen S2 (deformation scale factor: 50x)

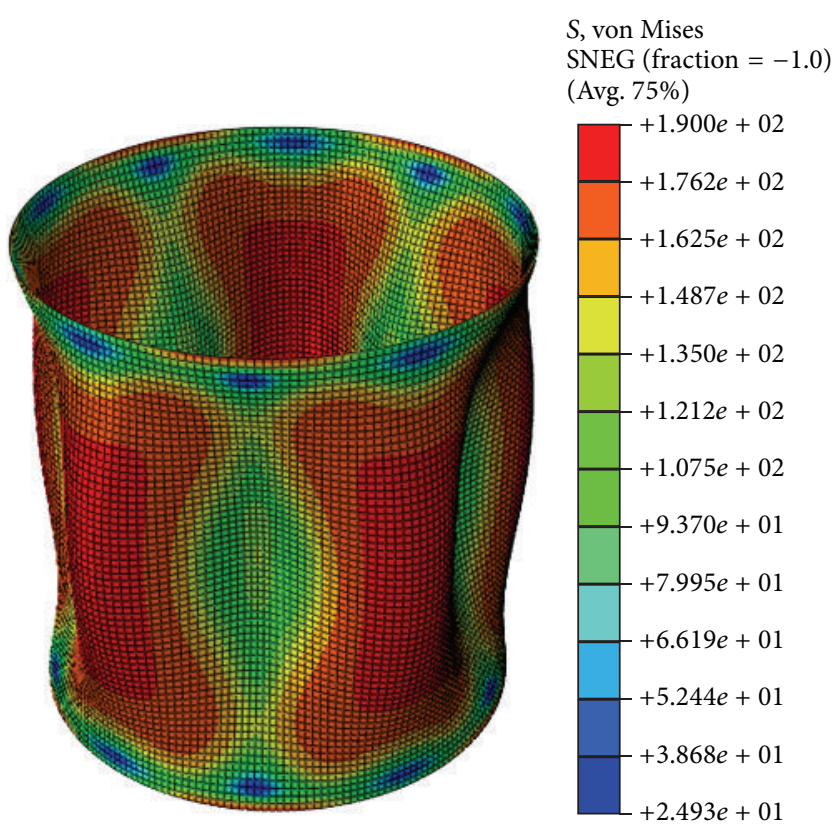

(b) Specimen SP.3-Set A (deformation scale factor: 20x)

FIGURE 4: 3D isometric views of the deformed shapes and von Mises stress contour plots at the ultimate pressure for specimen S2 (a) and specimen SP.3-Set A (b).

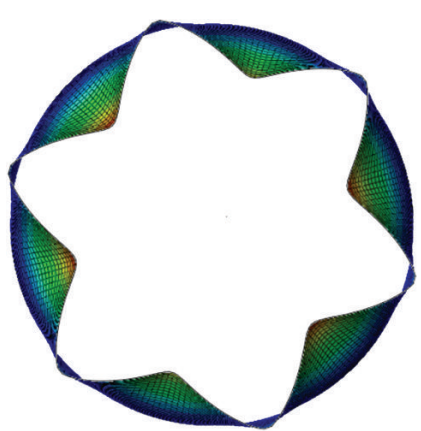

(a) Buckling shape by FE (ABAQUS) using either the flow or deformation theory

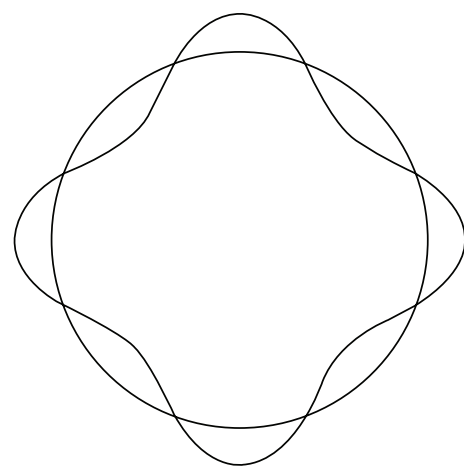

(b) Buckling shape by the analytical treatment using flow theory

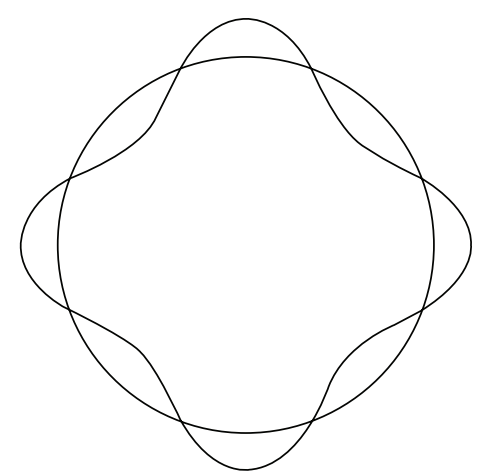

(c) Buckling shape by the analytical treatment using deformation theory

FIGURE 5: Specimen S2 [5]: comparison between buckling shapes from different methods.

in order to lead to the same value of the buckling pressure. This is the case, for example, of specimens S2 [5] and SP.3-Set A [6], as shown in Figures 4, 5, and 6.

Also this is not surprising, given that the kinematics in the $\mathrm{FE}$ approach is far less constrained than that in the analytical one [3].

Overall the presented investigation suggests that, also in the case of nonproportional loading, there is actually no plastic buckling paradox. In fact, when the buckling shapes coincide, the analytical treatment of the problem based on the flow theory of plasticity leads to predictions of the buckling load which are very close to the corresponding test results. This is again in contrast with the conclusions by other authors and with the widely accepted belief that the flow theory leads to a significant overestimation of the buckling stress while the deformation theory leads to much more accurate predictions.

The discrepancy between the results from the analytical use of the deformation or of the flow theory of plasticity arises instead when the buckling modes do not coincide. This can be related to the phenomenon of change in buckle patterns in elastic structures studied by Chilver [15], Supple [16], and Guarracino et al. [17-19], among others. The phenomenon can be attributed to an interference between the geometrical and material properties, which can induce a change of the collapsing modes. In such a case, it might happen that adopting the deformation theory of plasticity contributes to counterbalancing the enhanced stiffness induced by kinematically constraining the cylinders to follow 


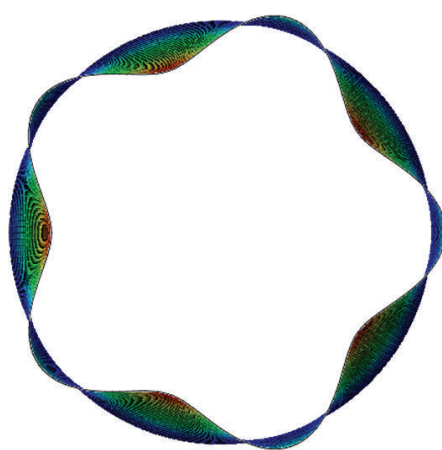

(a) Buckling shape by FE (ABAQUS) using either the flow or deformation theory

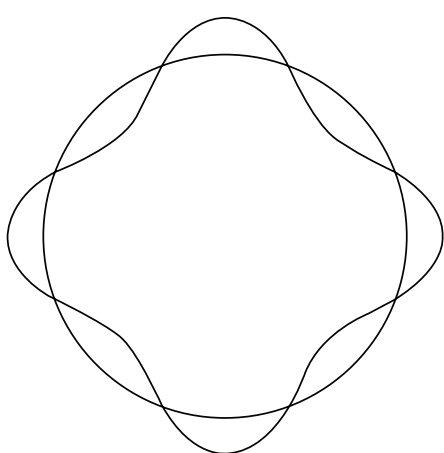

(b) Buckling shape by the analytical treatment using flow theory

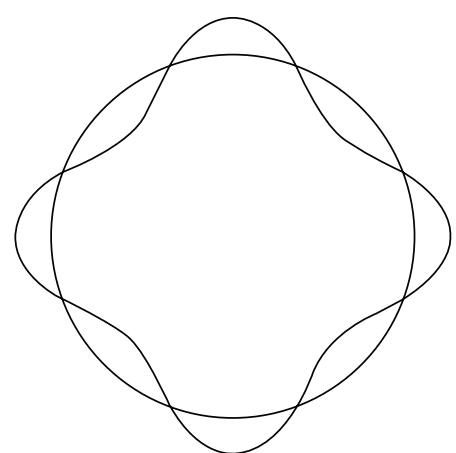

(c) Buckling shape by the analytical treatment using deformation theory

FIGURE 6: Specimen SP.3-Set A [6]: comparison between buckling shapes from different methods.

predefined buckling modes, thus providing results that are only apparently more in line with the experimental findings.

Once again, this does not happen in the case of carefully constructed and validated nonlinear FE analyses [8].

\section{Conclusions}

An analytical analysis of the plastic buckling of cylinders subjected to nonproportional loading, that is, axial tensile load and external pressure, has been presented with the aim of providing further understanding of the apparent discrepancy between the predictions of the flow and deformation theories of plasticity. By comparing the analytical results with experimental and numerical results, the following conclusions may be drawn:

(i) The numerical FE predictions based on the flow theory of plasticity [8] are in good agreement with the experimental results. This is in contrast to the conclusions by other authors that the flow theory, as in the case of BOSOR5 code results, leads to incorrect predictions of plastic strains and buckling pressures while the deformation theory leads to much more accurate predictions.

(ii) The buckling loads calculated analytically using both the flow and deformation theories of plasticity often match those obtained numerically by using the code BOSOR5.

(iii) The root of the apparent discrepancy seems to be a change in the buckling modes induced in some cases by the different material stiffness provided by the deformation and flow theories of plasticity. In such cases there is an apparent overestimation of the buckling loads by the flow theory while the deformation theory counterbalances the excessive kinematic stiffness of the cylinder due to a constrained kinematics.

The conclusion of the present investigation is that also in the case of cylinders subjected to nonproportional actually there seems to be no plastic buckling paradox.

\section{Conflict of Interests}

The authors declare that there is no conflict of interests regarding the publication of this paper.

\section{References}

[1] R. Hill, The Mathematical Theoy of Plasticity, Clarendon Press, Oxford University Press, New York, NY, USA, 1998.

[2] A. Mendelson, Plasticity: Theory and Application, Macmillan Company, New York, NY, USA, 1968.

[3] R. Shamass, G. Alfano, and F. Guarracino, "A numerical investigation into the plastic buckling paradox for circular cylindrical shells under axial compression," Engineering Structures, vol. 75, pp. 429-447, 2014.

[4] R. Shamass, G. Alfano, and F. Guarracino, "A numerical investigation into the plastic buckling of circular cylinders," in Shell Structures: Theory and Application, W. Pietraszkiewicz and J. Górski, Eds., vol. 3, pp. 441-444, CRC Press, New York, NY, USA, 2013.

[5] J. Blachut, G. D. Galletly, and S. James, "On the plastic buckling paradox for cylindrical shells," Proceedings of the Institution of Mechanical Engineers, Part C: Journal of Mechanical Engineering Science, vol. 210, no. 5, pp. 477-488, 1996.

[6] J. J. Giezen, C. D. Babcock, and J. Singer, "Plastic buckling of cylindrical shells under biaxial loading," Experimental Mechanics, vol. 31, no. 4, pp. 337-343, 1991.

[7] D. Bushnell, "BOSOR5-program for buckling of complex, branched shells of revolution including large deflections, plasticity and creep," in Structural Analysis Systems, A. Niku-Lari, Ed., vol. 2, pp. 25-54, Pergamon Press, New York, NY, USA, 1986.

[8] R. Shamass, G. Alfano, and F. Guarracino, "An investigation into the plastic buckling paradox for circular cylindrical shells under non-proportional loading," Thin-Walled Structures. In press.

[9] J. J. Giezen, Plastic buckling of cylinders under biaxial loading [Ph.D. thesis], GALCIT, California Institute of Technology, Pasadena, Calif, USA, 1988, SM 88-23.

[10] R. Peek, "An incrementally continuous deformation theory of plasticity with unloading," International Journal of Solids and Structures, vol. 37, no. 36, pp. 5009-5032, 2000. 
[11] H. Jahed, S. B. Lambert, and R. N. Dubey, "Total deformation theory for non-proportional loading," International Journal of Pressure Vessels and Piping, vol. 75, no. 8, pp. 633-642, 1998.

[12] J. Chakrabarty, Applied Plasticity, Springer, New York, NY, USA, 2nd edition, 2010.

[13] Simulia, ABAQUS Theory Manual. Version 6.11-1, Dassault Systems, Waltham, Mass, USA, 2011.

[14] J. C. Simo and T. J. R. Hughes, Computational Inelasticity, Springer, Berlin, Germany, 1998.

[15] A. H. Chilver, "Coupled modes of elastic buckling," Journal of the Mechanics and Physics of Solids, vol. 15, no. 1, pp. 15-28, 1967.

[16] W. J. Supple, "On the change in buckle pattern in elastic structures," International Journal of Mechanical Sciences, vol. 10, no. 9, pp. 737-745, 1968.

[17] F. Guarracino and A. C. Walker, "Some comments on the numerical analysis of plates and thin-walled structures," ThinWalled Structures, vol. 46, no. 7-9, pp. 975-980, 2008.

[18] M. Fraldi and F. Guarracino, "Towards an accurate assessment of UOE pipes under external pressure: effects of geometric imperfection and material inhomogeneity," Thin-Walled Structures, vol. 63, pp. 147-162, 2013.

[19] M. Fraldi and F. Guarracino, "Stability analysis of circular beams with mixed-mode imperfections under uniform lateral pressure," Advances in Mechanical Engineering, vol. 2014, Article ID 294507, 7 pages, 2014. 


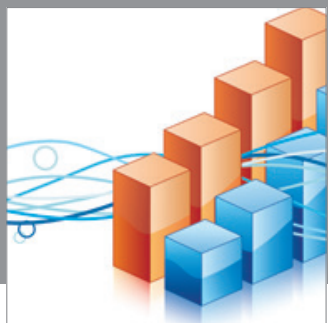

Advances in

Operations Research

mansans

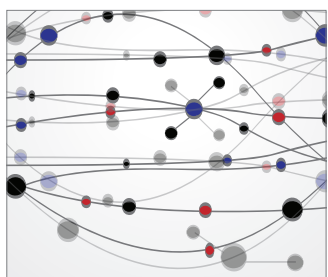

The Scientific World Journal
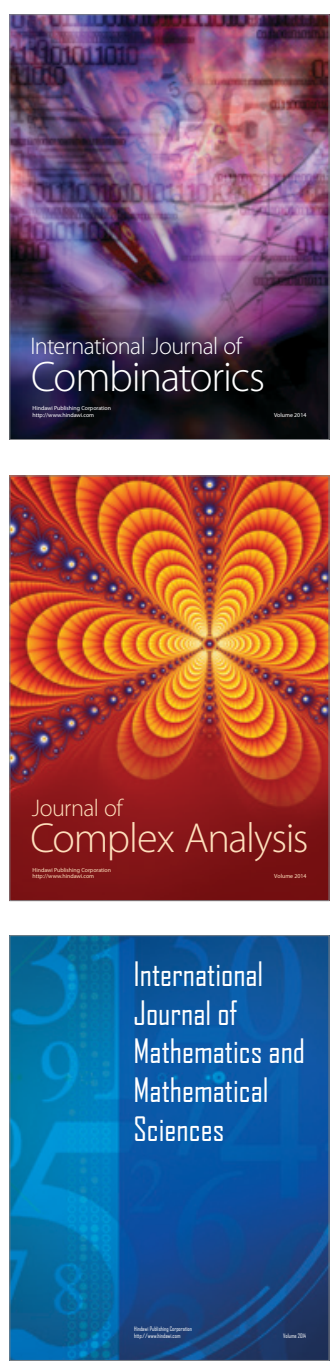
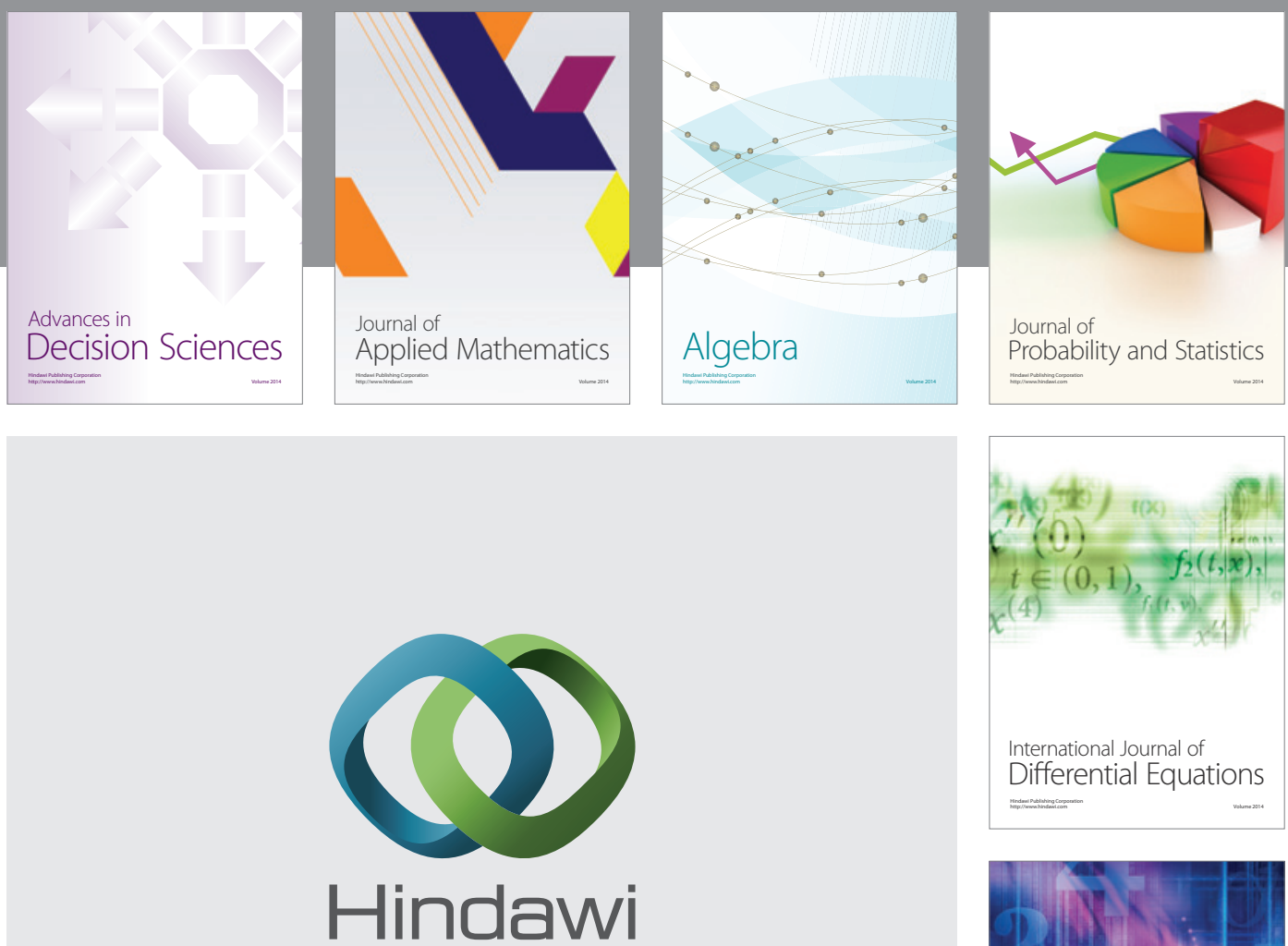

Submit your manuscripts at http://www.hindawi.com
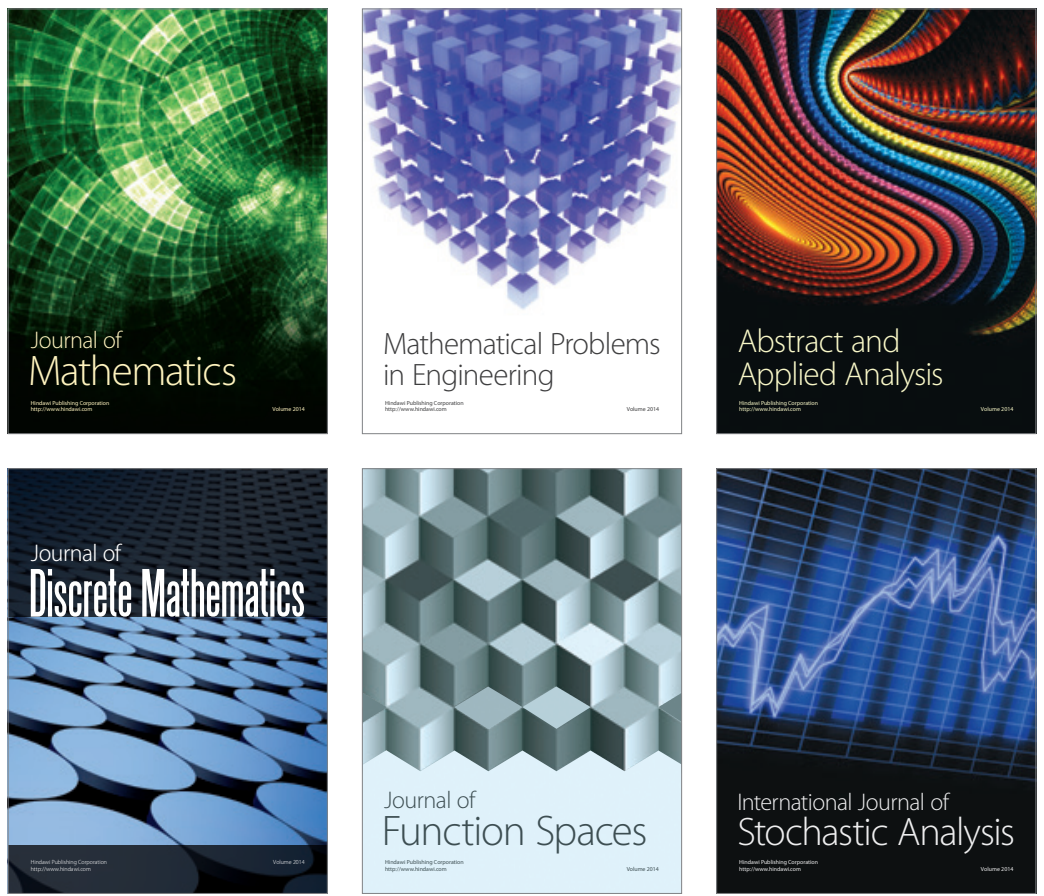

Journal of

Function Spaces

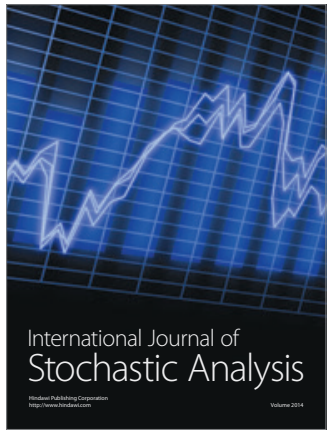

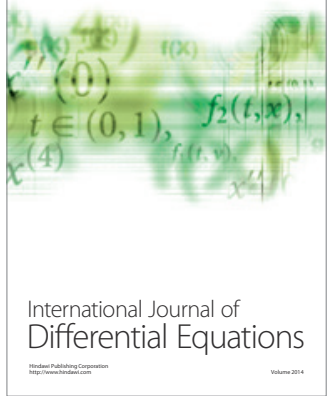
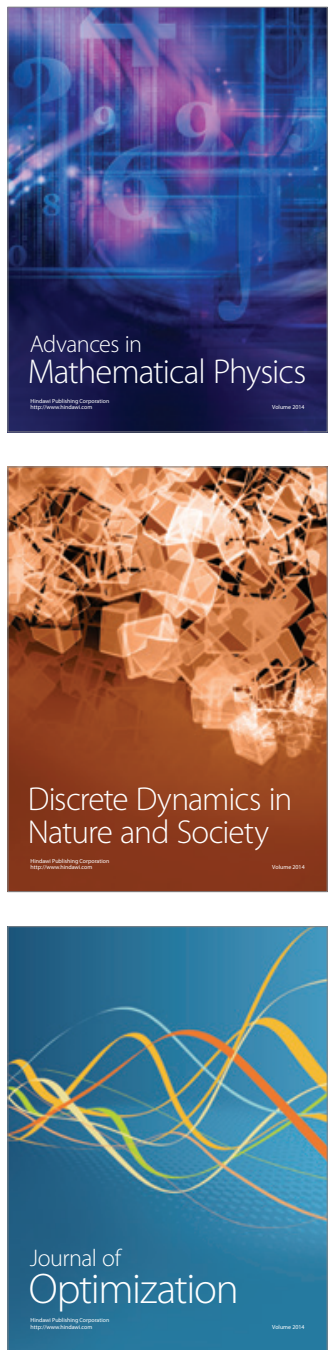